\title{
INTEGRAÇÃO ENSINO, PESQUISA E EXTENSÃO NA ENGENHARIA ATRAVÉS DA MOSTRA POLI
}

INTEGRATION TEACHING, RESEARCH AND EXTENSION IN ENGINEERING THROUGH

MOSTRA POLI

Alberto Casado Lordsleem Jr. ${ }^{1}$, Hiran Ferreira Lira ${ }^{2}$, Roberta Andrade de Araújo Fagundes ${ }^{3}$, José Roberto de Souza Cavalcanti ${ }^{4}$

DOI: 10.37702/REE2236-0158.v40p425-436.2021

\begin{abstract}
RESUMO
A extensão universitária é um dos pilares da educação superior brasileira, promovendo a interação transformadora entre as instituições de ensino e os outros setores da sociedade, por meio da produção e da aplicação do conhecimento, em articulação permanente com o ensino e a pesquisa. Os eventos de extensão são atividades voltadas à disseminação do conhecimento técnico-científico, constituindose uma importante ferramenta de integração. Nesse contexto, a Coordenação Setorial de Extensão e Cultura da Escola Politécnica da Universidade de Pernambuco promove a Mostra de Extensão, Pesquisa e Inovação (MOSTRA POLI). A avaliação é uma das mais importantes etapas do evento, pois permite conhecer e melhor utilizar os pontos fortes e eliminar ou adequar os pontos fracos. Nesse contexto, o presente artigo tem como objetivo apresentar a avaliação da MOSTRA POLI do ano de 2020 sob o ponto de vista dos participantes autores de trabalhos, comitê científico e comitê organizador. A metodologia contemplou o desenvolvimento do questionário de avaliação, a descrição das atividades de implantação e a análise dos resultados sobre a apreciação da MOSTRA POLI 2020. Os achados permitiram concluir que as principais oportunidades de melhoria correspondem ao aumento de prazo para a submissão e a avaliação dos trabalhos, enquanto os principais destaques foram as participações discente e docente e a publicação de anais com ISSN. Como contribuição, salienta-se a disseminação do conhecimento, o que promove um ambiente colaborativo entre os participantes do evento, contribuindo ainda para o aprimoramento e para novas iniciativas de ações análogas.
\end{abstract}

Palavras-chave: extensão universitária; evento; mostra Poli; engenharia.

\begin{abstract}
University extension is one of the pillars of Brazilian higher education, promoting transformative interaction between educational institutions and other society sectors, through the production and application of knowledge, in permanent articulation with teaching and research. Extension events are activities aimed at disseminating technical-scientific knowledge, constituting an important integration tool. In this context, the Extension and Culture Sectorial Coordination of the Polytechnic School of the Pernambuco University promotes the Extension, Research and Innovation Exhibition (MOSTRA POLI). The evaluation is one of the most important event steps, as it allows to know and better use the strengths and eliminate or adjust the weaknesses. In this context, this paper present the MOSTRA POLI 2020 evaluation from the view point of participating authors, scientific committee and organizing committee. The methodology included the development of the evaluation questionnaire, the description of the implementation activities and the results analysis of the

\footnotetext{
${ }^{1}$ Doutor pela USP, Coordenador Setorial de Extensão e Cultura da Escola Politécnica da UPE, acasado@poli.br

${ }^{2}$ Mestre pela PUC, Gerente de Extensão da Escola Politécnica da UPE, hiranferreira@poli.br

${ }^{3}$ Doutora pela UFPE, Gerente de Cultura da Escola Politécnica da UPE, roberta.fagundes@ upe.br

${ }^{4}$ Mestre pela USP, Diretor da Escola Politécnica da UPE, jrcavalcanti@ poli.br
} 
MOSTRA POLI 2020. The findings allowed us to conclude that the main opportunities for improvement correspond to the increase in the deadline for papers submission and evaluation; while, the main highlights were the students and professors participation and the proceedings publication with ISSN. As a contribution, the dissemination of knowledge is highlighted, promoting a collaborative environment among the event's participants, also contributing to the improvement and new initiatives of similar actions.

Keywords: university extension; event; mostra Poli; engineering.

\section{INTRODUÇÃO}

A extensão universitária surgiu na Inglaterra do século XIX com o propósito de abrir novos caminhos para a sociedade e de desenvolver a educação continuada. $\mathrm{Na}$ atualidade, a extensão é o principal instrumento utilizado para a materialização do compromisso social, a qual busca aproximar a universidade e a comunidade, proporcionando benefícios e conhecimentos para ambas as partes (RODRIGUES et al., 2013; GUERSOLA; CIRINO; STEINER, 2016; BARBOSA et al., 2019).

A dinâmica extensionista envolve modalidades que se diferenciam quanto à finalidade e às características, quais sejam: programa, projeto, curso, oficina, evento e prestação de serviço (COUTTS; ROBERTS, 2003; LORDSLEEM; LIRA; RABBANI, 2014; FRANZ; GARST; GAGNON, 2015; DWYER et al., 2017; UPE, 2021).

Particularmente, o evento consiste em uma organização, promoção ou atuação em uma programação, implicando apresentação pública - livre, ou para clientela específica - de difusão de conhecimento, processo ou produtos culturais, científicos ou tecnológicos, desenvolvida ou reconhecida pela instituição como fóruns, congressos, seminários, simpósios, musicais, teatro e outros (GONÇALVES et al., 2020; UPE, 2021).

Os eventos são ainda considerados ações de interesse acadêmico e pedagógico que podem ser de caráter esporádico, de cunho educativo, técnico, social, científico, esportivo e artístico, objetivando a divulgação, o desenvolvimento e a ampliação dos conhecimentos produzidos pela universidade
(RODRIGUES et al., 2013; CAPRIOLI; LOPES; SILVA, 2019).

Em especial, o evento é o lugar propício de mostra da extensão universitária, como atestam diversos autores (GOMES et al., 2017; BARRETO et al., 2019; OLIVEIRA; MAINARDI; BORGES, 2019; RODRIGUES JR. et al., 2020), sendo fundamental eixo formativo das universidades, no qual as trocas de experiências e a integração entre projetos e programas, entre ensino, extensão e pesquisa se tornam realidade.

Entre as mais importantes etapas de um evento (NIKOLIĆ et al., 2015; SANTANA et al., 2015; GOMES et al., 2017; SANTOS et al., 2018), a avaliação deve ser alvo sistemático de ação, pois ajuda a identificar as fragilidades a serem corrigidas e a celebrar o sucesso dos acertos, tornando-o cada vez mais bem sucedido.

Conforme enfatizam Santana et al. (2015), a experiência de construção com base em frequente avaliação e feedback resulta em contribuições à gestão da extensão e ao gerenciamento dos eventos de uma universidade.

Dentro do contexto deste trabalho, a Mostra de Extensão, Inovação e Pesquisa, ou simplesmente MOSTRA POLI, é considerada o principal evento anual da Escola Politécnica (POLI) - unidade mais antiga da Universidade de Pernambuco (UPE), criada em 1912.

A MOSTRA POLI promove um espaço singular de diálogo e troca de experiências de ensino, pesquisa e extensão desenvolvidas na instituição, a qual será descrita na sequência.

A MOSTRA POLI é um evento integrante da Semana Universitária da UPE, sob a organização da Coordenação Setorial de 
Extensão e Cultura (CSEC) da POLI. Tem como principal objetivo apresentar ao meio acadêmico e à sociedade os trabalhos desenvolvidos pelos corpos docente e discente da POLI, promovendo a troca de experiências entre os participantes (CSEC, 2021).

O evento foi criado em 2014 com periodicidade anual e as apresentações de trabalho são definidas conforme a modalidade (monitoria, trabalhos de conclusão de curso, extensão, iniciação científica, mestrado e doutorado).

Participam da MOSTRA POLI todos os cursos existentes na instituição, quais sejam: Engenharia Civil, Engenharia da Computação, Engenharia de Controle e Automação, Engenharia Elétrica Eletrônica, Engenharia Elétrica Eletrotécnica, Engenharia elétrica Telecomunicações, Engenharia Mecânica e Física de Materiais (POLI, 2021).

As seguintes fases são contempladas para a consecução da MOSTRA POLI:

a) fase 1 -submissão de resumos: trabalho a ser formatado conforme as diretrizes do template disponibilizado para download em específica homepage. $\mathrm{O}$ resumo não pode exceder duas páginas, incluindo: título, nomes dos autores (aluno, orientador e demais coautores) e referências;

b) fase 2 - avaliação dos resumos: realizada por três docentes da Escola Politécnica da UPE (comissão científica), permitindo a comissão organizadora confirmar a aceitação do resumo submetido;

c) fase 3 - submissão da versão final: submeter $\mathrm{o}$ resumo corrigido considerando-se as solicitações do comitê científico;

d) fase 4 - submissão da apresentação: elaborada conforme as diretrizes do template disponibilizado para download em específica homepage;

e) fase 5 - apresentação e avaliação dos trabalhos: realizada considerando-se a área do conhecimento com avaliação por três docentes da Escola Politécnica da UPE (comissão científica);

f) fase 6 - compilação da avaliação dos trabalhos: apropriação das notas fornecidas pelos avaliadores, resultando na definição dos trabalhos mais bem posicionados em cada modalidade.

Os trabalhos aprovados são reunidos nos anais (ISSN 2359-2249) do evento com publicação na Plataform \& Workflow by OJS/PKP (http://revistas.poli.br/index.php/anais/index).

A Figura 1 ilustra as capas dos anais da MOSTRA POLI de 2014 até 2019; enquanto a Figura 2 ilustra a capa dos anais da edição mais recente, realizada em 2020.

A Tabela 1 reúne os quantitativos de resumos aprovados e de membros dos comitês organizador e científico de cada ano do evento.

Cada resumo indicado na Tabela 1 está associado a um autor principal do trabalho submetido à MOSTRA POLI.

Ressalte-se que no período de sete anos o quantitativo de resumos aprovados obteve um crescimento de $238 \%$, enquanto a participação nos comitês variou em até $300 \%$ no comitê organizador e $385 \%$ no comitê científico.

Figura 1 - Anais da MOSTRA POLI 2014-2019

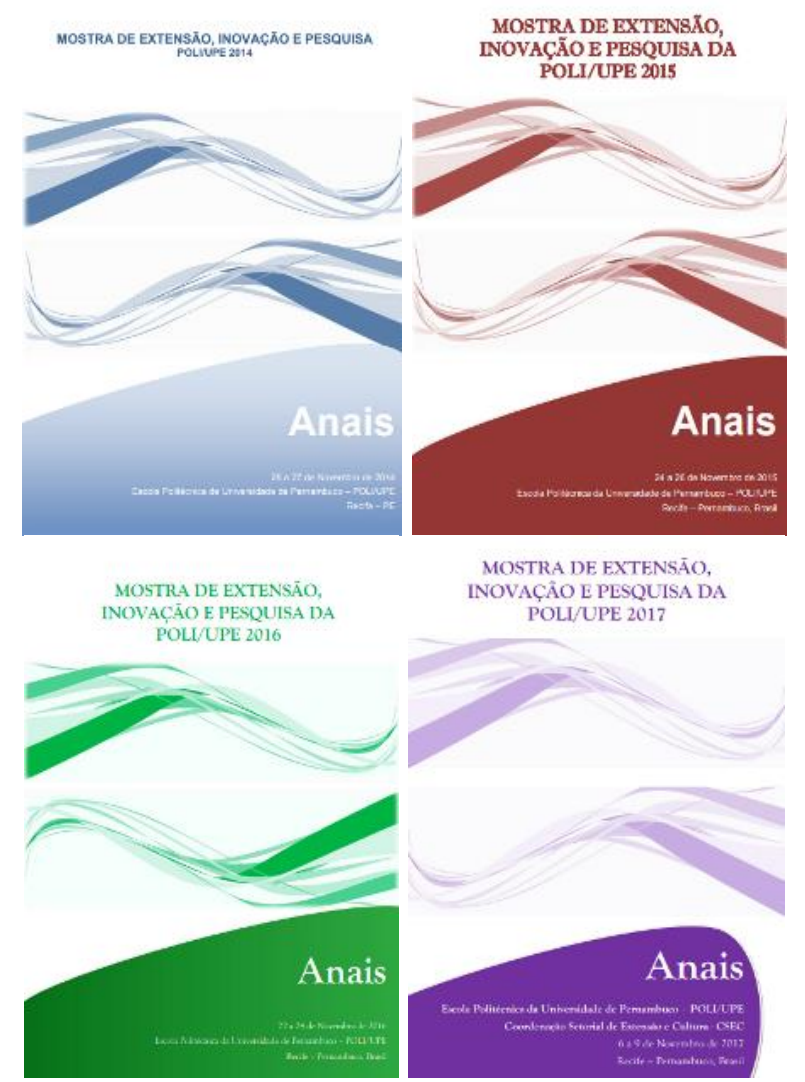




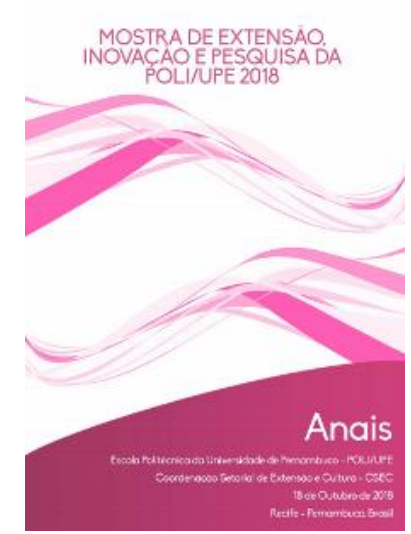

Fonte: acervo dos autores.

Figura 2 - Anais da MOSTRA POLI 2020

Fonte: acervo dos autores.

Tabela 1 - Quantitativos da MOSTRA POLI

\begin{tabular}{|c|c|c|c|c|}
\hline Ano & $\begin{array}{c}\text { Resumo } \\
\text { s } \\
\text { (autores } \\
\text { ) }\end{array}$ & $\begin{array}{c}\text { Comitê } \\
\text { organizado } \\
\mathbf{r}\end{array}$ & $\begin{array}{c}\text { Comitê } \\
\text { científic } \\
\mathbf{o}\end{array}$ & $\begin{array}{c}\text { Tota } \\
\mathbf{l}\end{array}$ \\
\hline 2014 & 50 & 6 & 21 & 77 \\
\hline 2015 & 57 & 10 & 13 & 80 \\
\hline 2016 & 93 & 13 & 22 & 128 \\
\hline 2017 & 90 & 15 & 32 & 137 \\
\hline 2018 & 101 & 18 & 50 & 169 \\
\hline 2019 & 107 & 9 & 38 & 154 \\
\hline 2020 & 119 & 16 & 39 & 174 \\
\hline Tota & 617 & 87 & 215 & 919 \\
$\mathbf{l}$ & & & & \\
\hline
\end{tabular}

Fonte: elaborada pelos autores.

\section{OBJETIVO}

Este artigo tem como objetivo principal apresentar e analisar a avaliação de satisfação da MOSTRA POLI realizada no ano de 2020, sob o ponto de vista dos participantes autores de trabalhos, comitê científico e comitê organizador.

\section{METODOLOGIA}

A metodologia utilizada contemplou pesquisa quantitativa descritiva da avaliação da satisfação, através de procedimento de levantamento de informações, baseado em questionários aplicados aos participantes da MOSTRA POLI 2020.

As seguintes etapas foram adotadas para o desenvolvimento deste trabalho, quais sejam:

a) etapa 1: revisão bibliográfica sobre o desenvolvimento de eventos de extensão e a importância no contexto universitário;

b) etapa 2: descrição da MOSTRA POLI no âmbito da Escola Politécnica da Universidade de Pernambuco;

c) etapa 3: o desenvolvimento do questionário de avaliação de satisfação dos principais intervenientes do evento MOSTRA POLI 2020;

d) etapa 4: a estruturação e a implementação da avaliação;

e) etapa 5: a coleta de dados e a análise dos resultados.

A seguir, são descritas as etapas 3, 4 e 5, tendo em vista que as etapas 1 e 2 foram apresentadas anteriormente.

\section{Desenvolvimento do questionário de avaliação}

O questionário foi desenvolvido com o objetivo de caracterizar os respondentes, avaliar a satisfação com os elementos do evento e prazos de atividades.

A caracterização dos respondentes contemplou quatro questões; os elementos foram avaliados em sete questões (duas questões específicas sobre prazos foram realizadas exclusivamente para os autores de trabalhos e para a comissão científica).

O Quadro 1 reúne os tópicos e itens constituintes do questionário, contemplando ainda a divisão das questões específicas realizadas aos autores de trabalhos e comitê científico. 
Quadro 1 - Parâmetros do questionário de avaliação da MOSTRA POLI 2020

\begin{tabular}{|c|c|c|}
\hline & \multirow{2}{*}{\multicolumn{2}{|c|}{ Itens }} \\
\hline Tópicos & & \\
\hline $\begin{array}{l}\text { Caracterização } \\
\text { dos } \\
\text { respondentes }\end{array}$ & \multicolumn{2}{|c|}{$\begin{array}{l}\text { - Origem } \\
\text { - Categoria de participação } \\
\text { - Conhecimento do evento } \\
\text { - Opção de realização do evento }\end{array}$} \\
\hline $\begin{array}{l}\text { Elementos } \\
\text { (pontos fortes e } \\
\text { fracos) }\end{array}$ & \multicolumn{2}{|c|}{$\begin{array}{l}\text { - Importância para a POLI } \\
\text { - Anais com ISSN } \\
\text { - Participação docente } \\
\text { - Participação discente } \\
\text { - Continuidade de participação } \\
\text { - Existência/organização } \\
\text { - Qualidade dos trabalhos }\end{array}$} \\
\hline \multirow[t]{2}{*}{ Específicos } & Autores & $\begin{array}{l}\text { - Prazo de submissão } \\
\text { - Prazo de correção } \\
\text { do resumo } \\
\text { (resubmissão) }\end{array}$ \\
\hline & $\begin{array}{c}\text { Comitê } \\
\text { Científico }\end{array}$ & 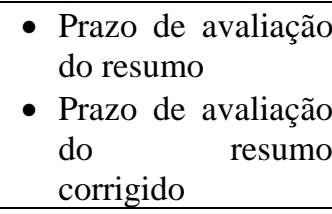 \\
\hline
\end{tabular}

Fonte: elaborado pelos autores.

Os itens questionados no tópico de caracterização dos respondentes correspondem às questões diretas. Para os itens dos tópicos de elementos específicos, as questões foram formuladas baseadas na escala Likert (MCLEOD, 2019).

A escala Likert é um método quantitativo usado em questionários para avaliar questões de acordo com o nível de concordância da audiência sobre as afirmações apresentadas, sendo assim denominada pois foi criada por Rensis Likert, sociólogo da Universidade de Michigan nos EUA (MCLEOD, 2019; PRADO, 2020).

A distribuição utilizada dos níveis da escala Likert foi a seguinte (Figura 3): concordo totalmente; concordo parcialmente; indiferente; discordo parcialmente; e discordo totalmente (MCLEOD, 2019; MACEDO, 2020; PRADO, 2020).

Figura 3 - Critérios da escala Likert na avaliação dos elementos

Concordo totalmente
Concordo parcialmente
Indiferente
Discordo parcialmente
Discordo totalmente

Fonte: acervo dos autores.
Em concordância com Macedo (2020), a adoção da escala Likert na pesquisa da MOSTRA POLI permitiu medir o grau de conformidade dos respondentes com as afirmações propostas, o nível de importância atribuída a uma atividade e, finalmente, a avaliação da atividade.

\section{Estruturação e implementação da avaliação}

A etapa de estruturação da avaliação iniciou com a parametrização do questionário da pesquisa em formulário no ambiente Google, conforme ilustra a Figura 4, com a mensagem inicial de introdução da pesquisa encaminhada na parte inicial do formulário

Figura 4 - Mensagem inicial no formulário Google sobre a pesquisa

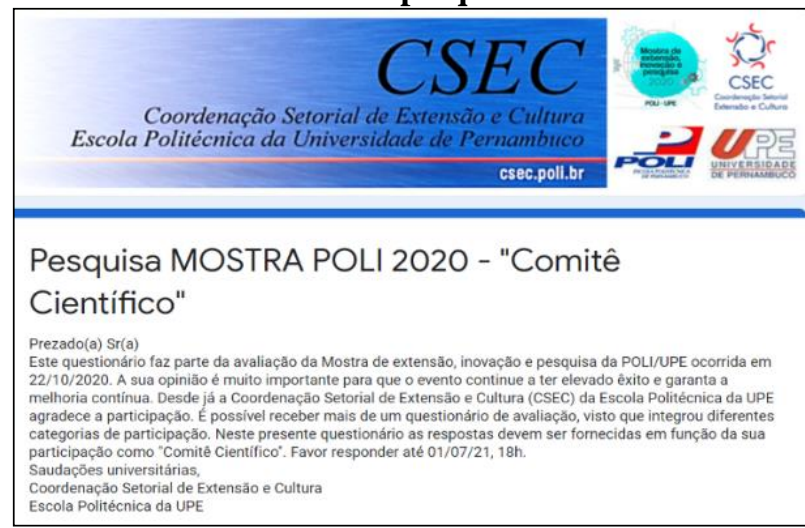

Fonte: acervo dos autores.

O Google Forms foi utilizado por ser gratuito, on-line, simples de usar, rápido para concluir e intuitivo. Além disso, permitiu organizar e analisar as informações para melhorar o compartilhamento e acesso.

A etapa de implementação da avaliação foi realizada com a configuração do Google Forms de envio de e-mail com o convite para preenchimento do formulário, a partir do cadastro de e-mails de autores de trabalho, comitês organizador e científico da coordenação da MOSTRA POLI.

As configurações estabelecidas no formulário demandaram resposta obrigatória, emitir recibo de resposta, limitar a uma resposta e mostrar barra de progresso. 


\section{A coleta de dados e análise dos resultados}

A etapa de coleta de dados foi realizada em julho de 2021, com o prazo de três dias para respostas. Ao final do prazo inicial, foram fornecidos mais dois dias de prazo final.

$O$ controle de recebimento de respostas foi programado por meio do FormLimiter-PROD (ferramenta suplementar que permite limitar a aplicação de um formulário através de configuração de data e hora, após os quais é encerrada a captura de respostas no Google Forms).

A análise de resultados contemplou a divisão por tópicos e itens para cada uma das categorias de participação, também comparação entre elas.

Os resultados e as principais considerações são a seguir apresentados.

\section{APRESENTAÇÃO E ANÁLISE DOS DADOS}

\section{Caracterização dos respondentes}

A integralidade dos respondentes, ou seja, $100 \%$ deles são filiados à POLI, seja como docente ou discente da instituição. Os autores apresentadores de trabalhos foram exclusivamente constituídos por discentes, assim como o comitê científico é constituído apenas por docentes. Já o comitê organizador foi constituído $79 \%$ por discentes e $21 \%$ por docentes.

A Tabela 2 apresenta os resultados de respondentes por categoria de participação. A coluna "\%" representa o percentual de respondentes em relação ao quantitativo total de participantes por categoria na MOSTRA POLI 2020 (conforme Tabela 1).

Tabela 2 - Parâmetros do questionário de avaliação da mostra poli 2020

\begin{tabular}{|l|c|c|}
\hline Categoria de participação & Respondentes & \% \\
\hline Autores & 42 & 35,3 \\
\hline Comitê Organizador & 7 & 43,8 \\
\hline Comitê Científico Total & 20 & 51,3 \\
\hline \multicolumn{2}{|r|}{ T9 } & 39,7 \\
\hline
\end{tabular}

Fonte: elaborada pelos autores.
A partir dos resultados mostrados na Tabela 2, verificou-se a participação, em ordem decrescente, do comitê científico, seguido do comitê organizador e autores. É possível associar o resultado àqueles participantes que dispõem de e-mail institucional da POLI/UPE de contato mais duradouro, visto que o comitê científico é formado por docentes, cuja permanência na universidade é mais longeva do que o comitê organizador e autores, majoritariamente formados por discentes, muitos dos quais já formados.

A Figura 5 ilustra os meios através dos quais os participantes tomaram conhecimento do evento; enquanto a Figura 6 ilustra a opção preferencial de realização do evento.

Figura 5 - Meios de conhecimento da MOSTRA POLI 2020

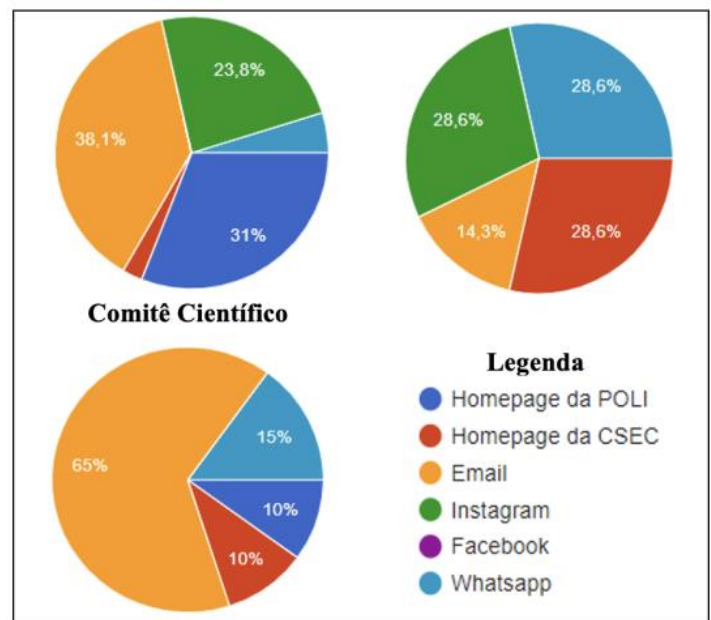

Fonte: elaborada pelos autores.

Figura 6 - opção preferencial de realização do evento

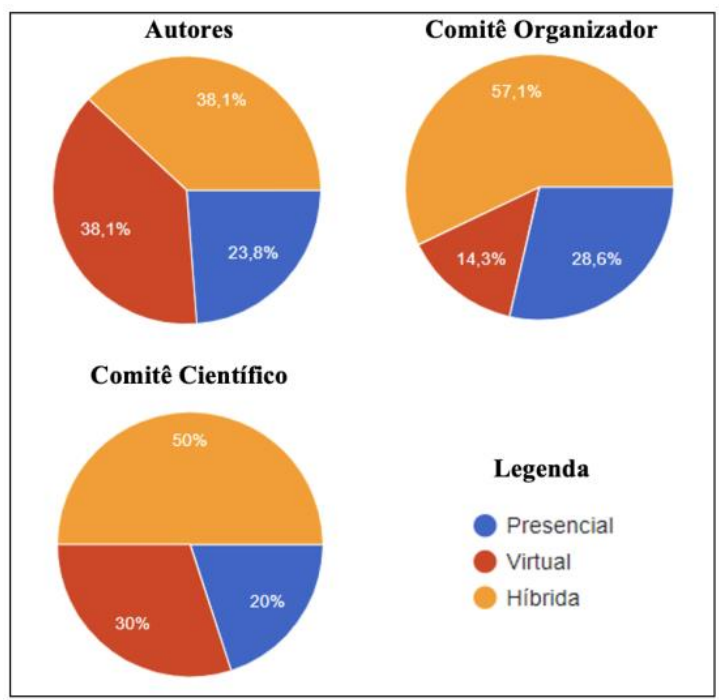

Fonte: elaborada pelos autores. 
De acordo com os resultados das Figuras 5 e 6, pode-se considerar:

a) o e-mail foi o principal meio de conhecimento da MOSTRA POLI 2020 pelo comitê científico $(65 \%)$ e autores apresentadores de trabalhos $(38,1 \%)$, diferentemente dos meios igualitários do comitê organizador (homepage da CSEC, Instagram e WhatsApp);

b) a opção híbrida $(48,4 \%)$ despontou como a principal na preferência para a realização da MOSTRA POLI 2020, enquanto a opção virtual foi de $27,5 \%$ e a presencial foi de $24,1 \%$.

O contexto atual de pandemia deve ter influenciado a opção pelo formato híbrido da MOSTRA POLI 2020, considerando o êxito das atividades virtuais, porém não descartando a opção da presença em conformidade com a atividade a ser realizada.

\section{Elementos}

As Figuras 7, 8, 9, 10, 11 e 12 reúnem os resultados da avaliação dos elementos (pertinentes às forças e fraquezas - ambiente interno) da MOSTRA POLI 2020.

De acordo com os resultados das Figuras 7 até 12, pode-se realizar as seguintes considerações:

a) a maioria $(95,2 \%)$ concorda que a MOSTRA é o principal evento técnico-científico anual da POLI, cuja ordem decrescente aponta a seguinte concordância: comitê organizador $(100 \%)$, comitê científico $(95 \%)$ e autores $(90,5 \%)$. Tal fato demonstra a importância da ocorrência do evento para a instituição;

b) entre os principais pontos fortes, em ordem decrescente, a maioria concorda como sendo a participação discente $(99,2 \%)$, empatando com 97,6\% a participação docente e a publicação de anais com ISSN. Destaque-se a concordância (100\%) quanto aos itens citados por parte dos comitês organizador e científico. Por outro lado, a participação discente $(88,7 \%)$ tem o maior percentual de concordância total em relação à participação docente $(68,5 \%)$ e publicação em anais $(66,9 \%)$;

c) a maioria $(95,9 \%)$ concorda quanto à satisfação pela existência e organização da MOSTRA POLI 2020. A média da concordância parcial $(37,9 \%)$ demonstra margem para atuação na melhoria desse item;

d) a maioria $(99,2 \%)$ concorda quanto à satisfação pela qualidade (conhecimento, clareza de exposição e conteúdo) dos trabalhos apresentados. A proximidade da concordância parcial dos autores apresentadores de trabalhos $(23,8 \%)$ e do comitê científico $(25 \%)$ demonstra sintonia entre os respondentes quanto à margem para atuação na melhoria desse item;

e) qualitativamente, percebe-se uma maior concordância total do comitê organizador em todos os itens avaliados, exceto pela existência/organização.

f) por outro lado, a maior quantidade de critérios utilizados encontra-se entre os autores.

Há concordância em $96 \%$ de participação contínua na MOSTRA POLI 2020, tendo apenas $11,9 \%$ dos autores de trabalhos demonstrado descontinuidade de participação. Pode-se supor que essa situação esteja alinhada com a formatura de alunos e término da graduação universitária.

\section{Específicos}

As Figuras 13 e 14 reúnem os resultados da avaliação dos tópicos específicos questionados aos autores dos trabalhos e comitê científico da MOSTRA POLI 2020, respectivamente.

De acordo com os resultados da Figura 13, pode-se realizar as seguintes considerações do ponto de vista dos autores: 
a) a maioria $(57,2 \%)$ concorda quanto ao prazo de duas semanas para a submissão do resumo, enquanto $30,9 \%$ apresentam discordância. Essa situação demanda análise do comitê organizador quanto à extensão de prazo;

b) a maioria $(71,4 \%)$ concorda quanto ao prazo de duas semanas para a correção do resumo, enquanto apenas $11,9 \%$ apresentam discordância.

Comparativamente com a discordância do prazo de submissão, a discordância do prazo de correção é bem menor, próximo ao terço.
De acordo com os resultados da Figura 14, pode-se realizar as seguintes considerações do ponto de vista do comitê científico:

a) a maioria $(90 \%)$ concorda quanto ao prazo de duas semanas para a avaliação do resumo, enquanto apenas $10 \%$ apresentam discordância;

b) a maioria (75\%) concorda quanto ao prazo de uma semana para a avaliação do resumo corrigido, enquanto $25 \%$ apresentam discordância. Essa situação demanda análise do comitê organizador quanto à extensão de prazo.

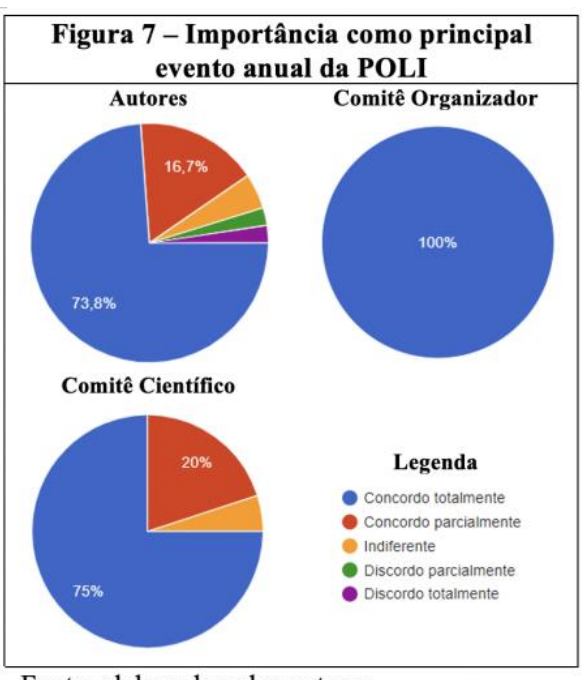

Fonte: elaborada pelos autores.

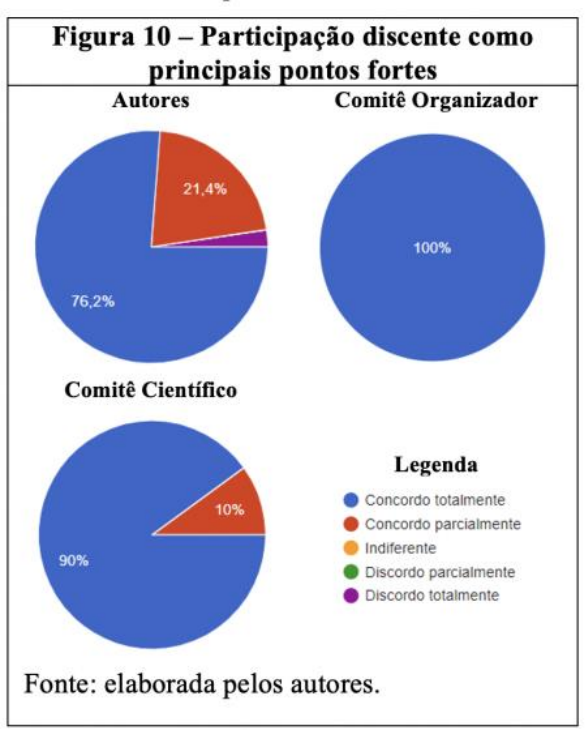

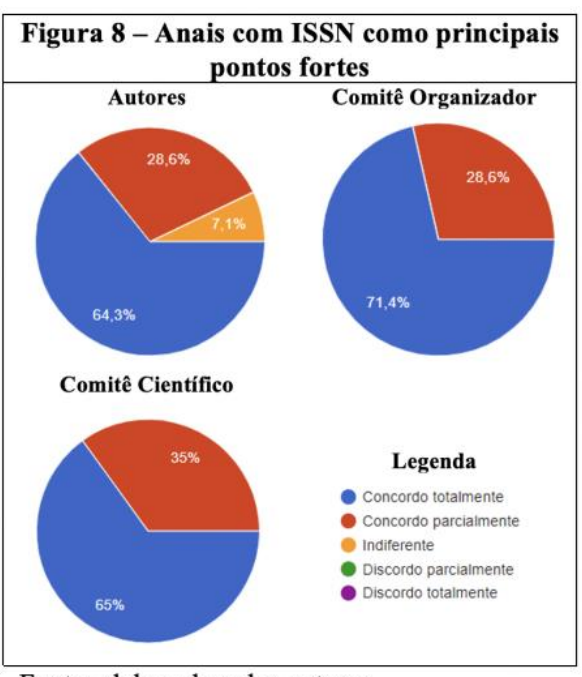

Fonte: elaborada pelos autores.

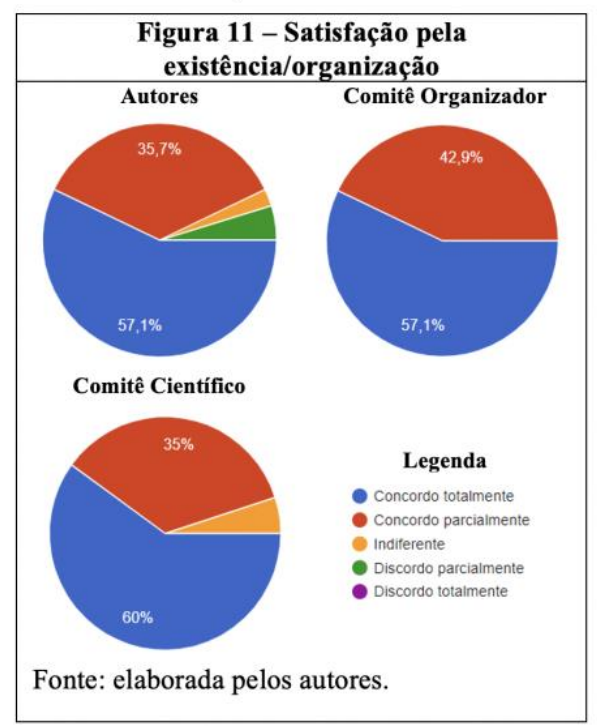

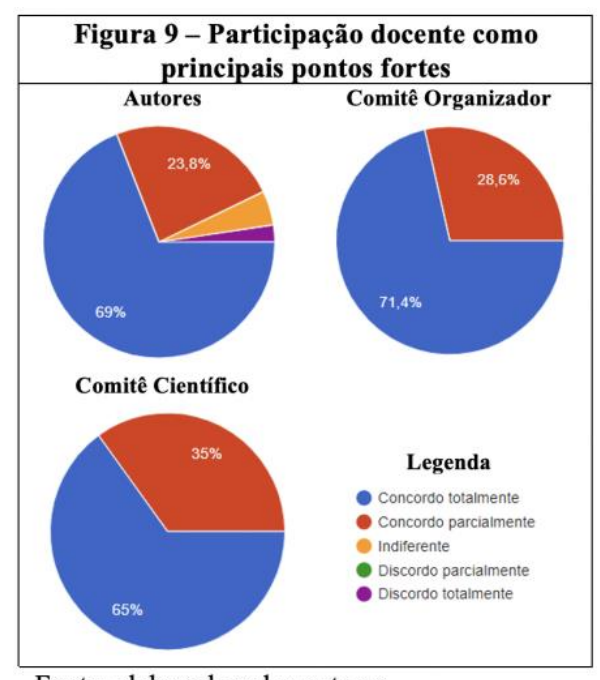

Fonte: elaborada pelos autores.

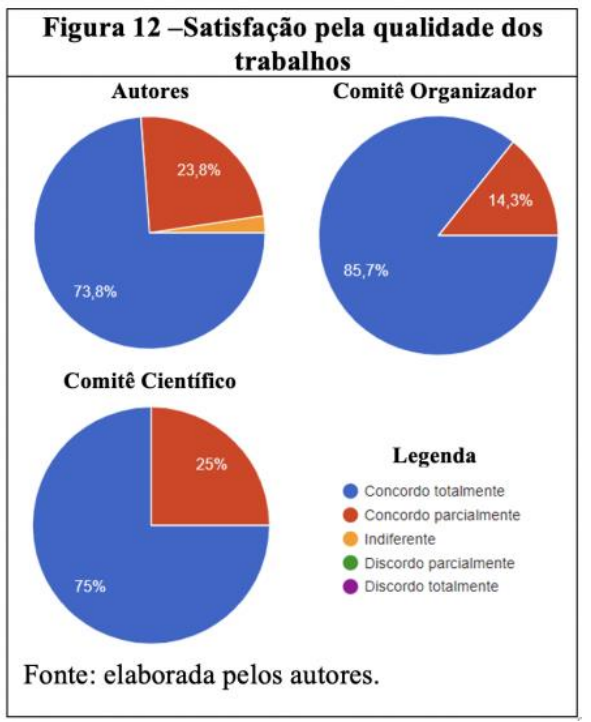




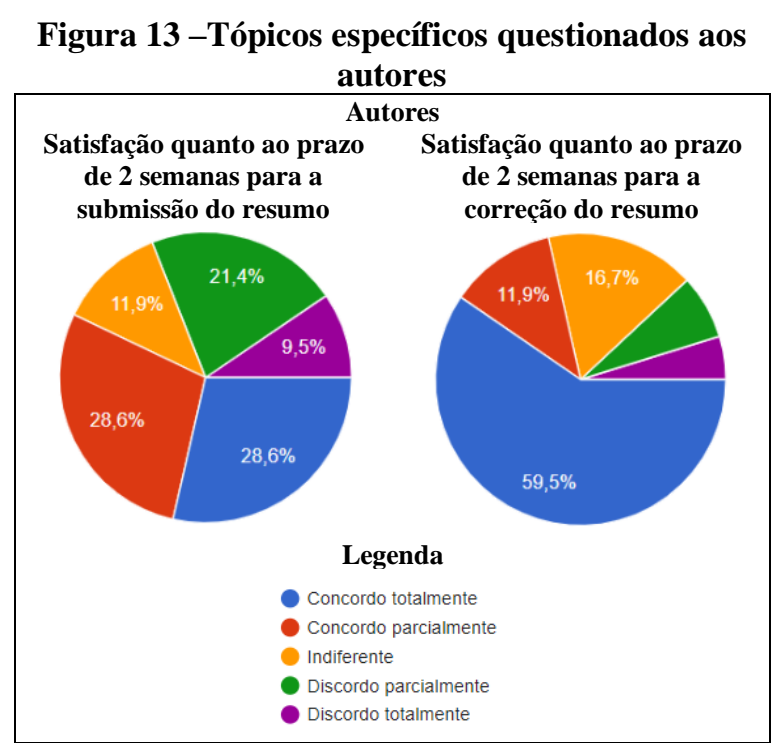

Fonte: elaborada pelos autores.

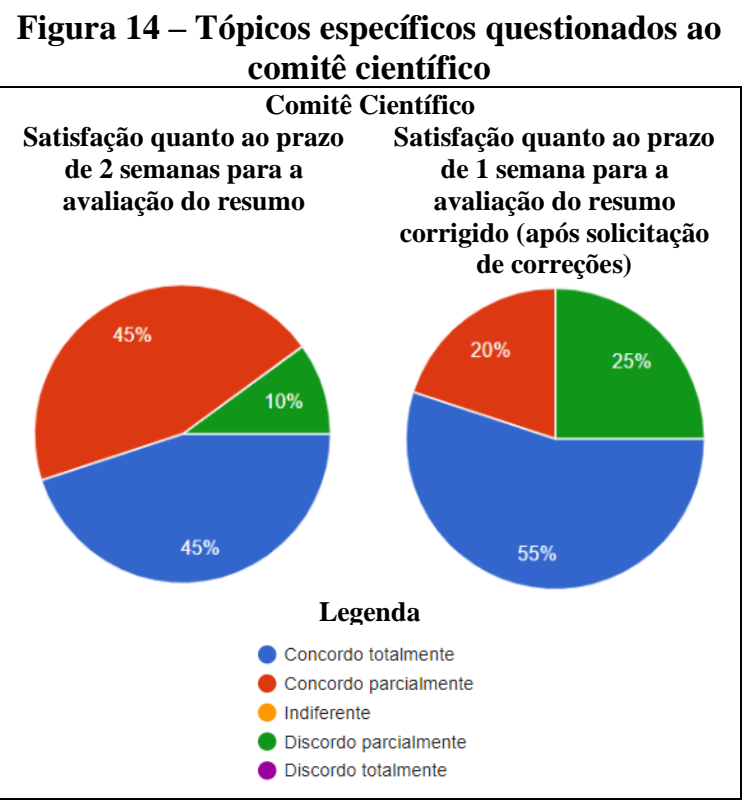

Fonte: elaborada pelos autores.

Comparativamente com a discordância do prazo de avaliação do resumo, a discordância quanto ao prazo de metade do tempo para a avaliação do resumo corrigido é 2,5 vezes superior.

\section{CONSIDERAÇÕES FINAIS}

Os respondentes (69) da pesquisa corresponderam a quase $40 \%$ do total de participantes (174), compreendidos entre autores, comitê organizador e comitê científico.

A partir dos resultados obtidos com a pesquisa de estudo de caso foi possível identificar as principais oportunidades de melhoria da MOSTRA POLI 2020, cujos itens (3) de menor percentual de concordância em ordem decrescente foram : o prazo de duas semanas para a submissão do resumo - junto aos autores; o prazo de uma semana para a avaliação do resumo corrigido (após a solicitação de correções) - junto ao comitê científico; e o prazo de duas semanas para a correção do resumo - junto ao comitê científico.

Os resultados também permitiram identificar os principais destaques (pontos fortes) da MOSTRA POLI 2020, cujos itens (3) de maior percentual de concordância em ordem decrescente foram: a participação discente; a participação docente; e a publicação de anais com ISSN.

Por fim, cabe ressaltar a contribuição deste trabalho quanto à avaliação da satisfação de eventos de extensão, apontando alternativas para a melhoria de encontros correlatos e, ainda, podendo ser reproduzido para análises semelhantes.

\section{REFERÊNCIAS}

BARBOSA, L. A. et al. Extensão como ferramenta de aproximação da universidade com o ensino médio. Caderno de Pesquisa, v. 49, n. 174, p. 316-327, 2019.

BARRETO, A. B. B. et al. Eventos científicos: uma ferramenta para instigar o conhecimento. 2019. Disponível em: $<$ https://moexp. osorio.ifrs.edu.br/anais/detalhe/1532>. Acesso em: 01 jun. 2021.

CAPRIOLI, A. B.; LOPES, R. C.; SILVA, J. F. Os eventos de extensão como ferramenta pedagógica em um polo de ensino a distância (EAD). Revista GETS, v. 2, n. 2, p. 164176, 2019.

\section{COORDENAÇÃO SETORIAL DE EXTENSÃO} E CULTURA (CSEC). Mostra POLI. 2021. Disponível em: <http://csec.poli.br/eventos/mostra-poli/>. Acesso em: 08 ago. 2021. 
COUTTS, J.; ROBERTS, K. Models and best practice in extension. Proceedings... APEN National Forum, Hobart, Tasmanian. Hobart: APEN, 2003.

DWYER, J. W. et al. Cooperative extension as a framework for health extension: the michigan state university model. Academic Medicine, v. 92, n. 10, p. 1416-1420, 2017.

FRANZ, N. K.; GARST, B. A.; GAGNON, R. J. The cooperative extension program development model: adapting to a changing context. Journal of Human Sciences and Extension, v. 3, n. 2, p. 3-12, 2015.

GOMES, M. A. B.; MICARONI, L.; MELLO, R. M. Q.; GUIMARÃES, J. L. Mostra de ciência: um evento de extensão da UFPR. Revista Extensão em Foco, n. 13, p. 121134, 2017.

GONÇALVES, A. K. C. et al. Extensão universitária no stricto sensu. Anais... Seminário de Extensão da PUC Minas 2020, Belo Horizonte: Extensão PUC Minas, 2020.

GUERSOLA, M. S.; CIRINO, P. D.; STEINER, M. T .A. Os papéis da universidade: uma visão dos discentes de engenharia de produção. Revista de Ensino de Engenharia, v. 35, n. 2, p. 44-53, 2016.

LORDSLEEM JR., A. C.; LIRA, H. F.; RABBANI, E. R. K. Coordenação setorial de extensão e cultura - estruturação, diagnóstico e propostas preliminares: relatório técnico. Recife: CSEC/POLI, 2014.

MACEDO, S. B. Quantos pontos são necessários? Um estudo comparativo de escalas likert, do tipo likert e semântica. Revista Horizontes Interdisciplinares da Gestão, v. 4, n. 2, p. 104-119, 2020.

MCLEOD, S.A. Likert scale definition, examples and analysis. 2019. Disponível em: $\quad<$ https://www.simply psychology.org/likert-scale.html>. Acesso em: 01 jun. 2019.

NIKOLIĆ, S. et al. A CERIF compatible CRISUNS model extension for assessment of conference papers. Acta Polytechnica Hungarica, v. 12, n. 7, pp. 129-148, 2015.

OLIVEIRA, G. T.; MAINARDI, C. F.; BORGES, G. R. Importância do desenvolvimento de eventos de extensão: um estudo de caso do SIMPAGRO da UNIPAMPA. Anais... $11^{\circ}$ Salão Internacional de Ensino, Pesquisa e Extensão da UNIPAMPA 2019, Bagé: UNIPAMA, 2019.

POLI. Ensino - graduação. 2021. Disponível em: <http://upe.poli.br/\#>. Acesso em: 04 ago. 2021.

PRADO, L. Escala Likert: entenda o que é e como utilizá-la. 2020. Disponível em: $<$ https://www.voitto.com.br/

blog/artigo/escala-likert>. Acesso em: 03 ago. 2020 .

RODRIGUES, A. L. L.; PRATA, M. S.; BATALHA, T. B. S.; AMARAL, C. L. N.; PASSOS NETO, I. F. Contribuições da extensão universitária na sociedade. Cadernos de Graduação - Ciências Humanas e Sociais, v. 1, n. 16, p. 141-148, 2013.

RODRIGUES JUNIOR, E. et al. Percepções de estudantes sobre uma mostra de experimentos de física realizada em um evento de extensão. Revista Extensão em Foco, n. 21, p. 143-155, 2020.

SANTANA, R. D. S. et al. Módulo para gerenciamento de eventos do SIEC - Sistema de Informação de Extensão e Cultura da UFG. Anais... Quinta Conferência de Directores de Tecnología de Información, TICAL 2015, Gestão de lasTIC's para la Investigación y la Colaboración, 2015, Vina del Mar: RedClara, 2015.

SANTOS, E. A. B. et al. Semana carranca - uma iniciativa complementar para o ensino da engenharia no atual contexto global. Anais... XLVI Congresso Brasileiro de Educação em Engenharia, Salvador, Brasil: COBENGE, 2018. 
UPE. UNIVERSIDADE DE PERNAMBUCO.

Resolução no 049, de 2021. Altera a política

de creditação da extensão na Universidade de

Pernambuco. CEPE, Recife, PE, jun. 2021.

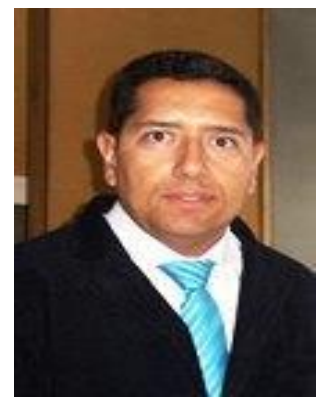

\section{DADOS BIOGRÁFICOS DOS AUTORES}

Alberto Casado Lordsleem Jr. - Graduação em Engenharia Civil pela UFPE (1994); Mestrado (1997), Doutorado (2002) e Pós-Doutorado (2010) em Engenharia de Construção Civil e Urbana pela Escola Politécnica da USP; Livre-Docência pela Universidade de Pernambuco (2012). Gerente de Pós-Graduação, Pesquisa e Extensão da Escola Politécnica da UPE (2007-2010). Atualmente é professor associado; coordenador setorial de extensão e cultura; docente permanente do Programa de Pós-Graduação em Engenharia Civil (PEC), docente de cursos de especialização e coordenador do MBA em Tecnologia e Gestão da Construção de Edifícios da Escola Politécnica da Universidade de Pernambuco. Coordenador do POLITECH - Grupo de Ensino, Pesquisa e Extensão em Tecnologia e Gestão da Construção de Edifícios. Assessor técnico de empresas e entidades setoriais da construção civil. Tem experiência na área de Engenharia Civil, com ênfase em Gestão da Construção, Desempenho e Inovação Tecnológica, atuando principalmente nos seguintes temas: construção civil, inovações tecnológicas, racionalização construtiva, desempenho, projeto para produção, vedações verticais, sistemas de gestão da construção, produtividade e recuperação de patologias.

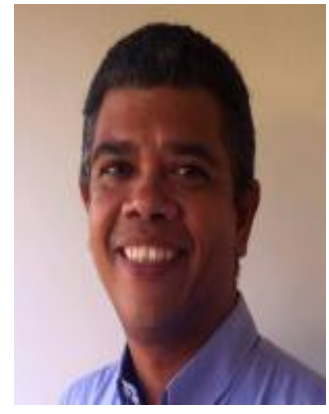

Hiran Ferreira Lira - Graduado em Licenciatura em Desenho e Plástica (atualmente - Licenciatura em Expressão Gráfica) pela Universidade Federal de Pernambuco (1990), especialista em Geometria Gráfica (1993) e Mestre em Design pela Pontifícia Universidade Católica do Rio de Janeiro (2003) e Doutorando no Programa de Pós-Graduação em Design na UFPE. Atualmente é professor em regime de dedicação exclusiva da Universidade de Pernambuco (POLI/UPE) e Gerente de Extensão na Coordenação Setorial de Extensão e Cultura. Suas áreas de interesse são: Expressão e Representação Gráfica, Geometria Gráfica Tridimensional, Educação, Design Educacional, Design de Recursos de Aprendizagem, EAD, Arte Sequencial, CAD, BIM, Modelagem e Impressão 3D.

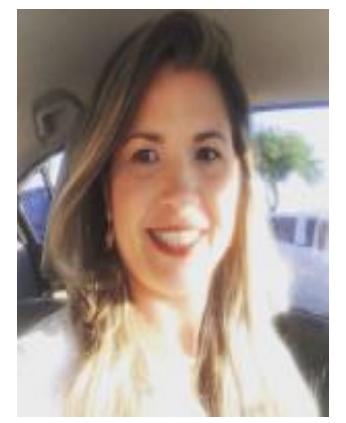

Roberta Andrade de Araújo Fagundes - Possui Pós-Doutorado em Estatística (2015) pela Universidade Federal de Pernambuco (UFPE). Possui também doutorado (2013) e mestrado (2006) em Ciência da Computação pela UFPE. Graduada em Tecnologia em Telemática (2002) pelo Centro Federal de Educação Tecnológica da Paraíba (CEFET-PB). Atualmente é Professora Adjunta da Universidade de Pernambuco (2007) do curso de Sistemas de Informação e de Engenharia da Computação na Universidade de Pernambuco (UPE). Também é vice coordenadora e professora do Programa de Pós-Graduação de Engenharia da Computação (PPGEC), onde há cursos de Mestrado e Doutorado. Possui interesse em pesquisa na área de Ciência da Computação, com ênfase em Inteligência Computacional. 
José Roberto de Souza Cavalcanti - Possui graduação em Engenharia Civil pela Universidade de Pernambuco (1984), graduação em Bacharelado em Física pela Universidade Federal de Pernambuco (1982) e mestrado em Engenharia Civil pela Universidade de São Paulo (2001). Atualmente é Diretor da Escola Politécnica da Universidade de Pernambuco, professor da Universidade de Pernambuco. Tem experiência na área de Física, Engenharia Civil e Segurança no Trabalho, atuando principalmente nos seguintes temas: conforto ambiental, conforto térmico, conforto, avaliação pós ocupacional e qualidade. 